\title{
A FAST LARGE-AREA POSITION-SENSITIVE TIME-OF-FLIGHT NEUTRON DETECTION SYSTEM ${ }^{*}$
}

\author{
R. K. Crawford and J. R. Haumann \\ Argonne National Laboratory
}

DISCLAIMER

\begin{abstract}
This report was prepared as an account of work sponsored by an agency of the United States Government. Neither the United States Government nor any zgency thereor, nor any of their employees, makes any warranty, express or implied, or assumes any legal liability or responsibiliry for the accuracy, completeness, or usefulness of any information, apparatus, product, or process disclosed, or represents that its use would not infringe privately owned rights. Reference herein to any specific commercial product, process, or service by trade name, trademark, manufacturer. or otherwise does not necessarily constitute or imply its endorsement, recommendation, or favoring by the United States Government or any agency thereof. The views and opinions of authors expressed herein do not necessarily state or reflect those of the United States Government or any agency thereor.
\end{abstract}

\section{Paper prepared for IEEE Nuclear Science Symposium}

\author{
held at San Francisco
}

October 25-27, 1989 


\title{
A FAST LARGE-AREA POSITION-SENSITTVE TIME-OF-FLIGHT NEUTRON DETECTION SYSTEM*
}

\author{
R. K. Crawford and J. R. Haumann \\ Argonne National Laboratory
}

Abstract

A new position-sensitive time-of-flight neutron detection and histograming system has been developed for use at the Intense Pulsed Neutron Source. Spatial resolution of roughly I $\mathrm{cm} \times 1 \mathrm{~cm}$ and time-of-flight resolution of $-1 \mu \mathrm{sec}$ are combined in a detection system which can ultimately be expanded to cover several square meters of active detector area. This system is based on the use of arrays of cylindrical one-dimensional position-sensitive proportional cointers, and is capable of collecting the $x-y-t$ data and sorting the. $m$ into histograms at time-averaged data rates up to $-300,000$ events/sec over the full detector area and with instantaneous data rates up to more than fifty times that. Numerous hardware features bave been incorporated to facilitate initial tuning of the position encoding. absolute calibration of the encoded positions, and automatic testing for drifts.

\section{INTRODUCTION}

A new neutron spectrometer, the Glass, Liquids, and Amorphous Materials Diffractometer[1] (GLAD) is being cnastructed at the Intense Pulsed Neutron Source (IPNS). This spectrometer will employ a polychromatic pulsed incident beam of thermal and epithermal neutrons, and will measure the sample scattering intensities as functions of scattering angle $2 \theta$ and wavelength $\lambda$ over a broad range of scattering angles $\left(2^{\circ}-90^{\circ}\right)$ and wavelengths $(0.1-4.0 \AA)$ in order to determine the scattering probabilities as functions of the momentum transfer $Q$ given by

$$
Q=\frac{4 \pi}{\lambda} \sin \theta
$$

Figure 1 shows the planned layout for GLAD. The time-offlight (TOF) principle will be used to detemine neutron wavelengths. Both instantaneous anã time-averaged data rates are expected to be relatively high (in extreme cases up to $-20,000,000$ events/sec instantaneous and up to $\sim 300,000$ events/sec time-averaged, when the full detector complement is installed). The resolution requirements dictate that the detection system for the thermal and epithermal neutrons must have spatial resolution approaching $1 \mathrm{~cm} \times 1 \mathrm{~cm}$, at least at the smaller scattering angles, and must have TOF resolution of -1

\footnotetext{
"Work supponted by U.S. Department of Energy, BES, contract No. W-31-109-ENG-38
}

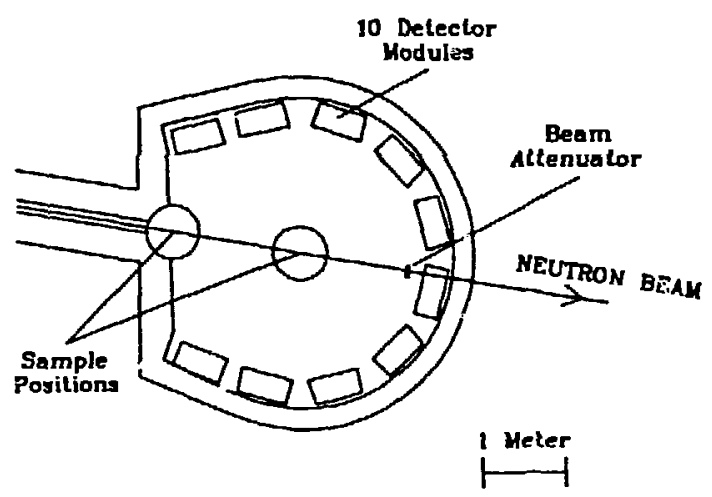

Figure 1. Plan view of the GLAD instrument.

usec. It is also necessary that the selected detection system be of a type which can ultimately be expanded to provide several $\mathrm{m}^{2}$ of active detector area at reasonable cost. Because this instrument will be operated in a closely-scheduled "user" mode, the detector system must be quite reliable and easily adjusted and calibrated. Finally, the detection system must be coupled with a data acquisition system which can sort the neutron events into $x-y-t$ histograms at the anticipated high data rates.

This paper describes the combined detection, positionencoding, and data acquisition system which was developed to meet the needs of GLAD. This system has incorporated a number of features to facilitate initial tuning of the position encoding, absolute calibration of the encoded positions, and automatic testing for drifts in calibration, and these will be discussed in some detail. A prototype version of the GLAD instrument has been in operation at IPNS for more than a year. Data obtained with this prototype has enabled verification of the performance capabilities of this detection/data-acquisition system, and these will also be discussed.

\section{DETECTORS AND POSITION ENCODING}

\section{Overview}

The system developed to meet these requirements is based on the use of detector modules, each of which contains an array of linear position-sensitive detectors (PSDs). Arrays of linear PSDs were chosen rather than two-dimensional PSDs, because the latter typically bave severe overall instantaneous 
data rate limitations. The number of $\mathrm{YSD}$ per module will vary somewhat depending on the module's location in the instrument, but is typically in the range of 40-60. In addition to the PSDs, each detector module incorporates a positioncalibration mechanism consisting of a neutron-absorbing bar which can be driven to known positions in front of the PSDs by a stepping-motor drive. The GLAD instrument will accommodate up to 19 such detector modules, as showa in Fig. 1. Figure 2 shows schematically a typicai layout of one such module. The neutron PSDs selected are cylindrical ${ }^{3} \mathrm{He}-$

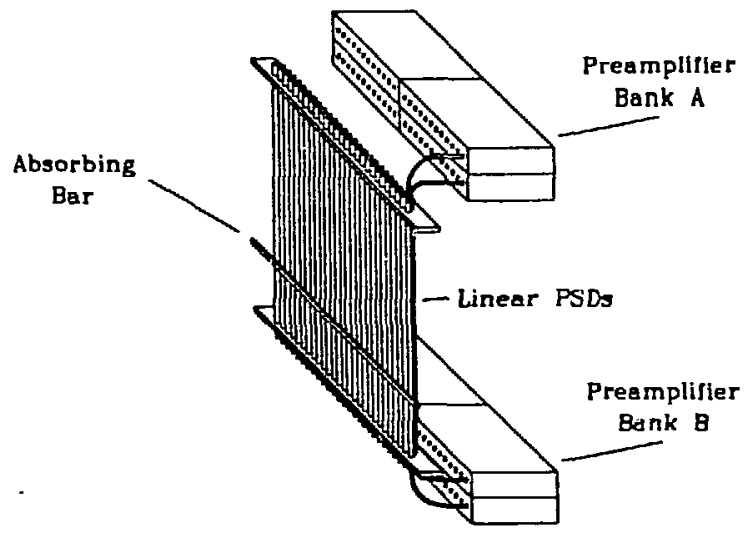

Figure 2. Schematic representation of a typical GLAD detector module, showing linear PSDs, preamplifiers, and the absorbing bar for position calibration. For clarity, only two of the sets of coaxial cables between the PSDs and preamplifiers are shown, and the drive mechanism for the absorbing bar is omitted from the drawing. filled gas proportional counters, exci $1.27 \mathrm{~cm}$ dia $\times 60 \mathrm{~cm}$ active length, filled with $10 \mathrm{~atm}$ of ${ }^{3} \mathrm{He}$ plus additional stopping and quench gases. The intrinsic spatial resolution due to the stopping range of this fill gas is $-0.5 \mathrm{~cm}$ fwhm, and the neutron detection efficiency is $\sim 0.56$ at $1 \AA$. Each end of each PSD is connected to its own charge-sensitive preamplifier, with total gains chosen to provide output pulses in the $0-1$ volt range. These output pulses are then transported over relatively long $(\sim 30 \mathrm{~m})$ coaxial cables to CAMAC Encoder modules, where the event positions (along individual PSDs) are digitized using charge-division encoding. Each Encoder module provides the position and TOF encoding for four such PSDs. The entire encoding chain is shown schematically in Fig. 3.

The neutron detection process starts with the absorption of a neutron at a distance $x$ from end $A$ of one particular PSD (Fig. 2). The absorption reaction is

$$
\mathrm{n}+{ }^{3} \mathrm{He}-{ }^{1} \mathrm{H}+{ }^{3} \mathrm{H}+765 \mathrm{KeV}
$$

The resulting proton and triton ionize the stopping-gas molecules, depositing the $765 \mathrm{KeV}$ of kinetic energy within a short distance $(-0.5 \mathrm{~cm})$ of the initial absorption. Gas amplification from the proportional mode operation then leads to a sizable charge being deposited on the anode wire at position $x^{\prime \prime}$, which is the projection on the anode of the center of the ionization cloud produced by the proton and triton (and so may differ from $x$ by up to $-0.5 \mathrm{~cm}$ for this fill-gas mixture). If $\mathrm{V}_{A}$ and $\mathrm{V}_{B}$ are respectively the peak voltages output by the charge-sensitive preamplifiers at ends $A$ and $B$

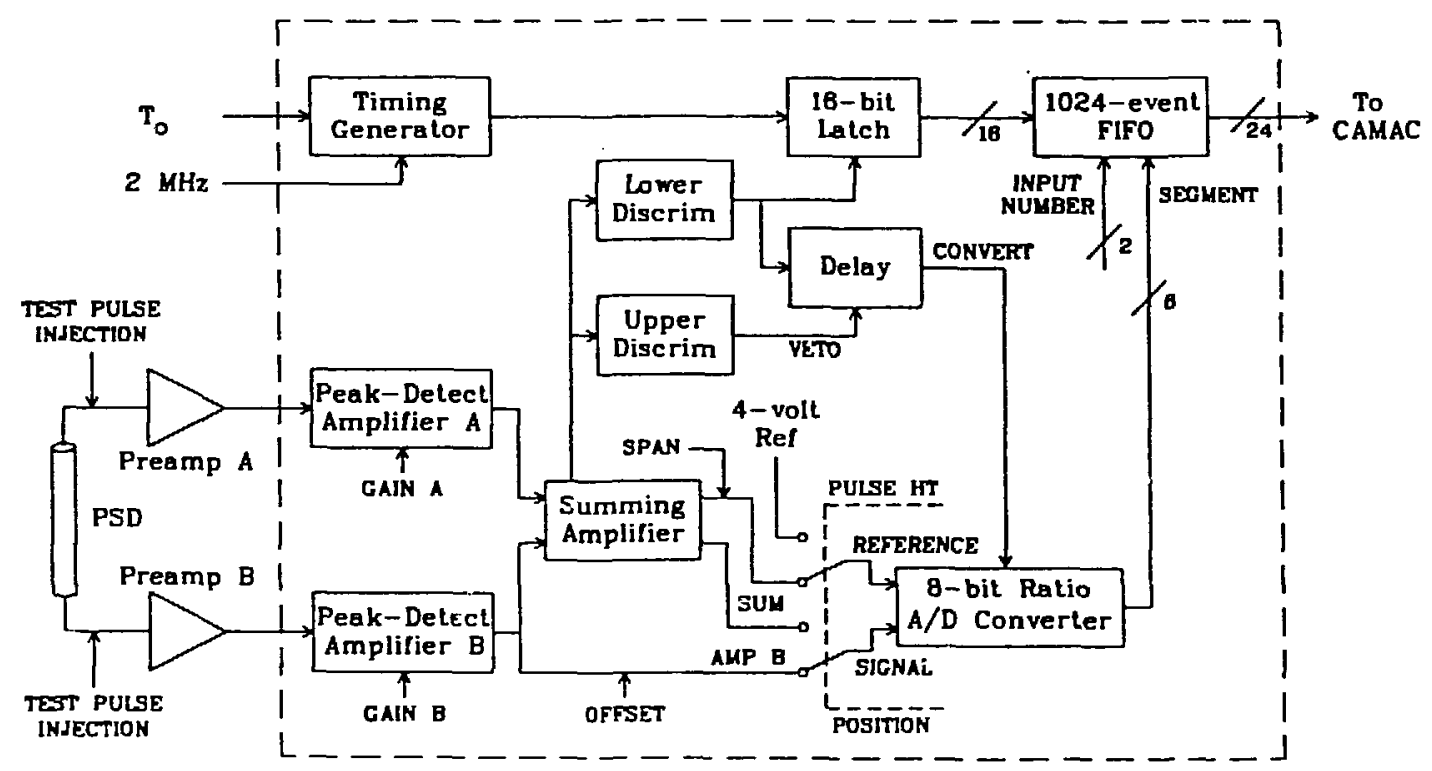

Figure 3. Schematic representation of the position encoding for one PSD at GLAD, including the PSD, preamplifiers, and one of four encodingpaths in a PSD Encoder module. (The portion inside the dashed box is incorporated in the Encoder module.) Also shown are the points at which test pulses from pulse generators can be injected for calibration purposes, and the "gain", "offset", and "span" adjustment points. The lower discriminator level is also adjustable. The Puise-Hu/Position switch, shown set in the Position mode, is software controlled. 
of the PSD of length $L$, then the encoded position $x^{\prime}$ is given by the charge-division equation

$$
\bar{L}=\frac{v_{B}}{v_{A}+V_{B}}
$$

In the final stage of the encoding, the quantity $x^{\prime} / L$ is digitized to provide a position segment or channel number in the range 0-63 for this event.

The linearity of the relationship between $x$ ' and $x$ " produced by such encoding is strongly influenced by the preamplifier gain, by other components in the loads terminating the two ends of the PSD, and by the timeconstants of the preamplifiers and amplifiers [2], but as will be shown below suitable choices can be made to provide encoding which is quite linear over the entire length of the PSD. Overall position resolution is determined by the intrinsic resolution associated with the proton and triton ranges in the fill gas, by the electronic noise in the analog portions of the encoding circuit (primarily in the preamplifiers), and by the stability and precision of the analog-to-digital conversion. (Several detailed treatments of resolution in charge-division encoding are available. $[3,4]$ ) Deadtime and recovery effects for such a system are on a PSD-by-PSD basis, with events in one PSD having no effect on the response of any other PSD to a subsequent event. The ability of a PSD and its encoding electronics to recover from an event before another occurs in the same PSD is govemed by the preamplifier and amplifier time-constants and linearities, and by the rime required for the analog-to-digital conversion.

\section{Preampiifiers}

The preamplifiers used at each end of the PSDs are a twostage charge-sensitive front-end design. The first stage is made up of a monolithic low-noise charge/current pulse preamplifier chip connected in a charge-sensitive configuration. It produces an output current proportional to the input charge and has an integration time-constant of -1.3 $\mu$ sec. This is followed by a second stage of current-to-voltage gain using a high-speed monolithic opamp chip. The gain of these preamplifiers is selected to produce output voltage pulses in the range of $0-1$ volts with 1200 volts applied to the PSD ancules.

These preamplifiers are constructed on $3.18 \mathrm{~cm} \times 7.6 \mathrm{~cm}$ printed circuit boards and mounted in modular housings containing 14 preamplifiers each. These modules also coniain the high voltage distribution and filtering for each PSD, as well as the test-pulse signal distribution to each r.reamplifier. The preamplifier housing modules are mounted as close as possible to the PSDs, and connected to each PSD through 30 cm long coaxial cables (Fig. 2).

\section{CAMAC Encoder Modules}

The output pulses from the preamplifiers are transmitted through conxial cables to the CAMAC PSD Encoder modules, which are located with the rest of the data acquisition electronics and the instrument computer some distance $(-30$ m) away from the preamplifiers and PSDs. Each Encoder module uses a standard CAMAC interface design, and incorporates independent encoding paths for four different PSDs. One such encoding path is shown schematically in Fig. 3. Separate peak-detecting amplifier stages are used for the signals from the two ends of the PSD, and the gains of these are independeotly adjustable to allow the inputs to be balanced and to provide an overall gain to match the pulse-heights of neutron-related events to the $0-4 \mathrm{~V}$ range of the analog-todigital encoder. Lower- and upper-level pulse-height discrimination is built into the system to minimize the counting of spurious events. The upper-level is fixed at $4 \mathrm{~V}$, but the lower-level for each encoding path can be adjusted to eliminate most eveats from gamma rays or fast neutrons. Additional "offset" and "span" controls on each encoding path allow fine-tuning of the mapping between the event positicn $x$ and the position-channel in which it is encoded, independently for each PSD. High-speed ratiometric analog-to-digital converter chips, one for each PSD, are used to provide rapid encoding. The precision limits of available inexpensive ratiometric converter chips ( 0.5 bit in 8 bits) result in roughly \pm 10 -percent variation in the widths of the encoded (6-bit) position-channels, but this does not pose a serious problem and is accounted for in the calibration process. For each event, the Encoder module also generates 16 bits of TOF information from a $2 \mathrm{MHz}$ master TOF Clock signal. The full event, each event at this stage being described by the 8 bits of detector element identification (encoding 64 position segments along each of four PSDs) plus the 16 bits of TOF, is then stored in a built-in first-in-first-out (FIFO) event buffer. Each Encoder module contains its own such FIFO with a depth of 1024 events. As long as any events are stored in the module FIFO the look-at-me (LAM) signal for that module is raised to signal the Auxiliary Crate Controller (see below) to transfer an event from this module.

The encoding of an event by one of these Encoder modules occupies only the PSD producing the event and the encodingpath associated with that PSD, and requires a fixed deadime on that encoding-path of $8 \mu \mathrm{sec}$ (deadtime is fixed at a constant value to simplify deadtime corrections in the data analysis). Since each PSD is thus completely independent as far as deadtime is concerned the full detection system can handle quite high instantaneous data rates without significant deadtime losses. However for GLAD the $8 \mu$ sec deadtime per event would still lead to an unacceptably high -40 percent deadtime correction at the projected maximum instantaneous rate of $\sim 50,000$ events per sec per PSD. Instantaneous rates approaching this maximum are expected to occur only in rare cases, and then only over a limited time range, but even so, some additional development efforts will be directed toward reducing this encoding deadtime. 
The Encoder modules can also be set by a softwarecontrolled switch to collect data in a 64-channel pulse-height mode for each PSD, rather than the usual 64-position mode (see Fig. 3). This feature allows automatic measurement of pulse-height spectra concurrently from all PSDs, and is used extensively in the initial adjustments of the Encoder modules and in the monitoring of the subsequent drifts of individual PSDs and encoding-paths, as discussed belorv.

\section{DATA TRANSFER AND HISTOGRAMING}

\section{System Overview}

A new FAST Data Acquisition System (FASTDAS) has been developed at IPNS to bandle the high instantaneous and time-averaged data rates expected for GLAD. This system is capable of histograming data in a fashion similar to the present microprocessor-based data acquisition systems $[5,6]$ in use for some years on other instruments at IPNS, but can build the histograms at a $300 \mathrm{KHz}$ time-averaged rate. An overview of the system is sketched in Fig. 4, and individual components are shown schematically in Figs. 5-6 and discussed separately below. This system begins with the digitized events in the

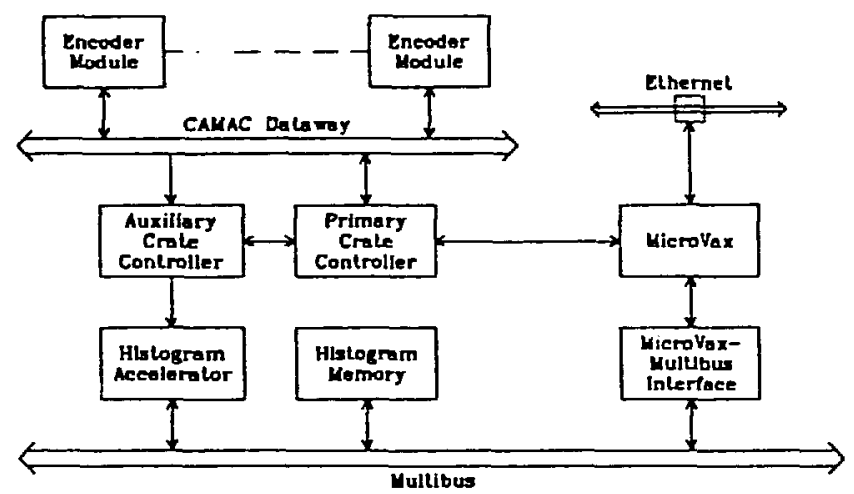

Figure 4. Block diagram of the FASTDAS data acquisition system.

FIFO buffers of the CAMAC PSD Encoder modules discussed above. These encoded events are then moved to a large FIFO buffer in a special CAMAC Auxiliary Crate Controller module. This Auxiliary Crate Controller feeds the events as fast as they can be accepted to a custom-designed Histogram Accelerator board located in a Multibus system, which histograms these events in predefined bins in a Multibus memory array. A microVax computer is directly coupled to the Multibus system by an intelligent Multibus interface and to each CAMAC crate by a commercial RS232 CAMAC Primary Crate Controller, and serves as the user interface to the system as well as providing on-line data analysis capabilities. This computer is networked to other computers to permit easy transfer of the data or analyzed results.
Each CAMAC crate can include up to 20 PSD Encoder modules, along with its Crate Controller, Auxiliary Crate Controller, and Master TOF Clock module. Up to 8 such crates can feed a single Multibus system with its Histogram Accelerator board and histogram memory boards, so the system car accommodate up to 640 linear PSDs encoded into a total of 40,960 position segments.

Data are histogramed in the form of different TOF spectra for the different position elements. Each position element can be assigned to its individual spectrum, or several elements can be assigned to the same spectrum ("zrouped") so that data from these elements are combined on-the-fly, All position elements are treated on an equal footing in the general binning algorithm, with no regard to the PSD on which they originate (the PSD associated with a given element can, of course, be considered when the initial grouping assignments are made). The range of TOF corresponding to a given channel in a particular TOF spectrum can be chosen to be any multiple of the fundamental TOF clock period ( $0.5 \mu \mathrm{sec}$ for GLAD), and every channel can have a different TOF width if so desired. A number of different TOF binning formats (specification of the TOF values corresponding to each channel in the spectrum) can be used concurrently, with one such format being used for each spectrum. Typically only a few different binning formats are defined, with each format being used for a number of different spectra. This histograming scheme is very similar to that used on the other IPNS instruments, which utilize multiple-microprocessor-based histograming systems.[5,6]

Three other features provided by the IPNS multiplemicroprocessor systems have also been built into FASTDAS. Multiple histograming of each event is permitted, so that each event can be binned in several different spectra, each according to its own independent element grouping and TOF format. (The produci of elements-per-histogram and the number of concurrent histograms is restricted to a maximum of 65,536 .) Anothur feature is a "new-histogram" option which allows rapid switching between histograms, permitting the real-time evolution of the spectra to be observed. The third feature is the provision for on-the-fly time-focusing, which allows events at TOF $t$ from element $i$ to be assigned a "focused TOF" $t_{i}^{*}$, and then binned into a spectrum according to $t^{*}$ rather than $t$. The focused TOF is computed event-by. event on-the-fly according to the algorithm

$$
t_{i}^{*}=c_{i}\left(t-t_{0}\right) \text {, }
$$

where $i$ runs over all detector elements and the focusing constants $c_{i}$ can be different for each element, while $t_{0}$ is a constant which is independent of detector element. Such timefocusing is currently used on several of the other IPNS instruments to allow events from different elements to be grouped according to the neutron momentum transfer $Q$ (Eq. 1 ), where $\theta$ depends on the detector element and $\lambda$ depends on the TOF $t$ and on the total flight path followed by the neutron in traveling from the source to the element $i$. On still other instruments Eq. 4 is used to focus according to neutron energy 
transfer, which depends on the distance from the sample to the detector element and on the TOF $t$. Focusing in either of these cases, as well as many others, can be achieved by appropriate choice of the set of constants $\left(c_{i}\right)$ and the constant $t_{0}$ to relate the desired physical quantity to the instrument geomery.

Although the FASTDAS system was developed for use with the PSDs in the GLAD instrument, it can readily be adapted to other instruments with other types of detectors. Such an adaptation requires the use of different Encoder modules appropriate to the detectors in question, but requires no changes in the remainder of the hardware system shown in Fig. 4. The FASTDAS system was designed with sufficient generality to meet the binning algorithm requirements of all IPNS instruments, so that other instruments could take

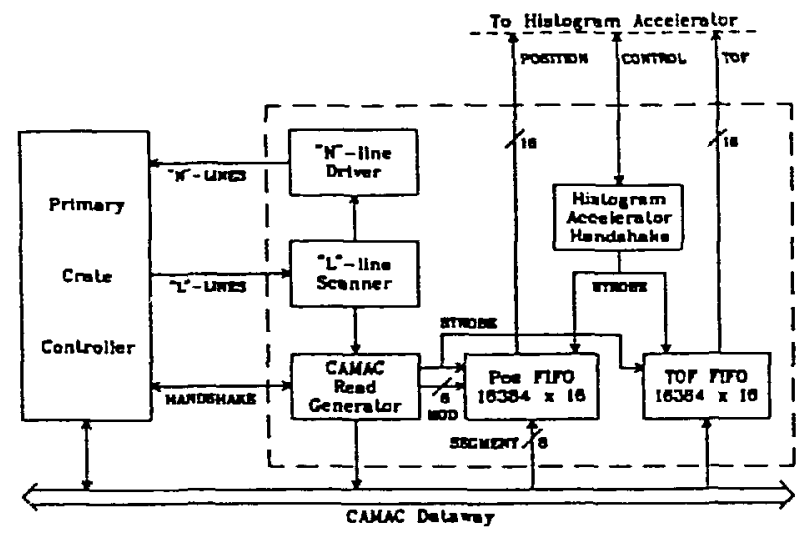

Figure 5. Block diagram of the FASTDAS CAMAC Auxiliary Crate Controller, showing its interactions with the Primary Crate Controller and with the Histogram Acceleralor. (The portions within the dashed box are contained within the Auxiliary Crate Controller.) advantage of the high speed offered by FASTDAS merely by duplicating the hardware and changing the binning tables downloaded to the Histogram Accelerator.

\section{Auxiliary Crate Controller}

The specially designed Auxiliary Crate Controller module (Fig. 5) scans the LAM lines from the 20 Encoder modules within the CAMAC crate at a $1 \mathrm{MHz}$ rate. When it finds a LAM set the Controller transfers one 24-bit ever:t from the Encoder module FIFO, combines this with the crate and slot number of this Encoder module, and stores the resulting 32-bit event ( 16 bits of position and 16 bits of TOF) in its own internal FIFO. This internal FIFO has a depth of 16,384 32-bit events. One such event is supplied from the Controller FFO to the Histogram Accelerator whenever requested by the latter.

The maximum rate at which the Auxiliary Crate Controller can remove events from a particular Encoder module is one event per scan. Since the Encoder module is scanned once every $20 \mu \mathrm{sec}$, the average data rate per Encoder module is limited to $50 \mathrm{KHz}$ or 1666 events per IPNS pulse $(30 \mathrm{~Hz}$ pulsing frequency). Since each Encoder module contains a 1024-event FIFO the peak data rate per module can be much higher for bursts of up to 1024 events before any events are lost due to buffer overflow.

\section{Histogram Accelerator}

The FASTDAS Histogram Accelerator (Fig. 6) is a single Multibus board which contains fast RAM-based lookup tables, adders, a multiplier, and read-increment-write logic for the Multibus memory. All RAM memory on this board is loadable from the microVax computer through the Multibus

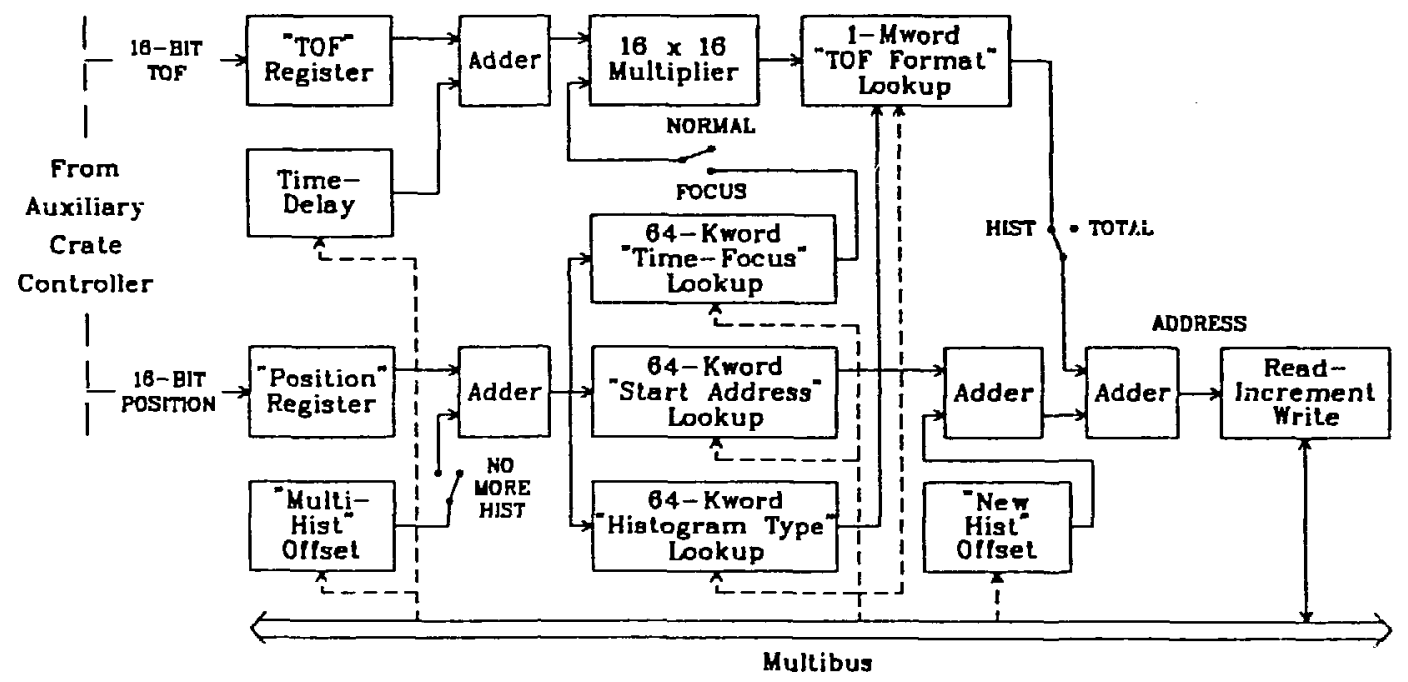

Figure 6. Block diagram of the FASTDAS Histogram Accelerator. Dashed lines show paths for lookup table and control registi: data downloaded from the microVax computer via the Multibus. 
address space. Using the configuration shown in Fig. 6 the Histogram Accelerator can duplicate nearly all the features of the multiple-microprocessor-based histograming system presently in operation on other instruments at IPNS, as outlined in the overview above, while histograming data at ten times the rate possible with the multiple-microprocessor systems.

At the star: of a histograming cycle, the Histogram Accelerator requests a 32-bit event from the CAMAC Auxiliary Crate Controller FIFO. The 16 bits of position element identification in this event address a $64 \mathrm{~K}$ word lookup table ("Start Address" table) which contains the "starting address" of the TOF spictrum for that detector element. This "starting address" is used as the upper 16 bits of a 24-bit spectrum address, so each TOF spectrum must start on a 256byte boundary in histogram memory. The 16 bits of position also address a 64-K word lookup table ("Histogram Type" table) which provides an index to one of up to sixteen different binning formats from the TOF-binning ("TOF Format") table. This histogram type table also contains flags indicating whether data from this position element are to be binned or ignored, whether each event from this element is to be binned in more than one histogram, and whether "on-the-fly time focusing" is to be applied to events from this element. More than one detector element can be assigned the same "starting address", allowing complete flexibility in the on-thefly combining or "grouping" of data from many elements. If the multiple-histograming option is in effect, then when this event has been histogramed in one spectrum the Histogram Accelerator returns to this point with the same 16-bits of position and 16-bits of TOF, but this time adds a "Multi-Hist" offset to the position data and repeats the binning process.

The 16 bits of TOF data first bas a constant $t_{0}$ added to it (contents of the "Time-Delay" register, which are zero if no constant time shift is desired), and if time-focusing is enabled this result is multiplied by a constant $c_{i}$ obtained from a 64Kword lookup table ("Time-Focus" table) addressed by position element. (If focusing is not enabled, the multiplication is by 1 instead.) The resulting focused TOF is then used to address the TOF Format lookup table which contains the various TOF binning formats, using $64 \mathrm{Kw}$ ords per format and up to 16 formats. Each format in this table provides a channel offset number for each 16-bit focused TOF value input to it. This tumber is to be added to the "starting address" for the TOF spectrum for this element to determine the address of the channel for binning this event. A value of zero for this TOF in this format table indicates that the TOF is out of range and therefore the event should not be binned. This scheme provides complete flexibility in TOF-binning, permitting the mapping of the raw or focused TOF information to varying numbers of channels per TOFspectrum and different channel widths at different TOF values.

If the event is to be histogramed, the Histogram memory address for the start of the TOF-spectrum for this event is taken to be the sum of the starting address (from the lookup table) for this element and the contents of the "New Hist" Offset register, all multiplied by 256 . The value in the New Hist Offset register is usually zero, but can be set to a new value by the microVax whenever desired, thus permitting rapid switching of the binning of all events to a different region of Histogram memory. In this way the data can be placed in several different histograms sequentially in order to follow the time-evolution of the scattering from the sample. To bin the event, the Histogram Accelerator first increments the contents of the 32-bit totalizing counter for the TOFspectrum (which is at the the TOF-spectrum starting address just computed). Then it also increments the contents of the channel at the add.zess calculated by adding to this spectrum starting address the channel offset address obtained from the TOF Format table. These channel offsets are chosen so that the TOF spectrum immediately follows the totalizing counter in Histogram memory. A jumper-selectable option allows the histograming to occur either in 16-bit channels or in 32-bit chanuels. In the 16-bit case, any overflows (channel "wraparound" due to more than 65,535 events per channei) which occur because of these increments will be recorded and attached to the data set when it is read out by the microVax. The use of a separate on-the-fly totalizing counter for each TOF-spectrum provides a convenient set of checksums permanently imbedded in the data, so that data integrity can be easily checked at any subsequent stage during the acquisition or later analysis.

Once the Histogram Accelerator has completed all required histograming for this event it immediately requests another event from the Auxiliary Crate Controller FIFO. The extensive FIFO buffering in the Encoder moduies and in the Auxiliary Crate Controllers completely derandomizes the incoming data, so the Histogram Accelerator is the controlling factor in the overall histograming rate. The governing factor is the Multibus memory incrementing done by the Histogram Accelerator, which is limited mainly by the Multibus arbitration time and the memory cycle time of the Multibus memory used. With the current $400 \mathrm{nS}$ cycle time memories, a time-averaged histograming rate of over $300 \mathrm{KHz}$ has been measured.

\section{Multibus System}

The Multibus, Multibus memory, and microVax-toMultibus interface are the same type as currently used in the other IPNS data acquisition systems, $[5,6]$ with only switchselectable modifications required to convert between systems. The Multibus has 24 memory address lines which provide an addressing capability of 16 Mbytes ( 1 "Mbyte" is really $2^{20}$ or $1,048,576$ bytes). Nearly 1.5 Mbytes of this are needed to address the Histogram Accelerator lookup tables (nearly 3 Mbytes are used for these tables, but since word-addressing is used on the Histogram Accelerator board this requires only 1.5 Mbytes of Multibus "address space"), thereby leaving 14.5 Mbytes available for addressing histogram data. Multibus memory boards can be added as required up to this 14.5 Mbyte 
limit to provide the physical storage necessary for whatever size histograms are planned (up to a maximum of 7.25 million 16-bit channels). All Multibus memory, including the lookup tables on the Histogram Accelerator board, is accessible to the microVax via the microVax-to-Multiuus interface which can address all 24 bits of address space.[5]

\section{MicroVax-to-CAMAC Interface}

The FASTDAS Histogram Accelerator issues no commands to the CAMAC system. Instead, the necessary initialization and control of the CAMAC modules is provided directly from the microVax through an RS-232 CAMAC Primary Crate Controller. This is quite simple and reliable, and is adequate since there are no speed-dependent functions which must occur via this link.

\section{ADJUSTMENT, CALIBRATION, AND MONITORING}

Overview

With such a large number of PSDs, efficient adjustment and calibration procedures are very important, as are the associated hardware and software features to facilitate calibration and to ensure the reliability of this calibration. Previous experience with linear PSDs at the University of Missouri[7] and at IPNS has been of great help in determining what adjustments must be done or checked and with what frequency, allowing the necessary hardware and software features to be incorporated in the present system at the outset. Two such hardware features, the absorbing-bar position calibration mechanism which is an integral part of each detector module, and the pulse-beight data acquisition mode for the FSD Encoder modules, have already been noted above. In addition, separate power supplies have been provided for each upper (A) and lower (B) bank of preamplifiers, allowing either bank in any detector medule to be tursed on or off independently and remotely, and test-pulse input points are connected to each A and B preamplifier allowing programmable tail-pulse generators to inject test pulses of known amplitudes independently into either the $A$ or $B$ (or both) banks of preamplifiers. These hardware capabilities and the associated software have been incorporated into the procedures developed for PSD adjustment, calibration, and monitoring, which are discussed below.

\section{Initiai Adjustment Procedure for CAMAC PSD Encoder Modules}

Whenever a new PSD, preamplifier, or PSD Encoder module is instailed, various adjustmeuts must be made in the associated encoding-path electronics to ensure linearity of the encoding and to match the position encoding to that of the other PSDs. The procedure described bere allows the necessary adjustments to be made relatively quickly whenever such a change is made, but need be repeated only infrequently otherwise. These steps can be followed concurrently for any number of PSDs. (All adjustments noted here are via potentiometers accessible through the front panels of the single-width CAMAC Encoder modules.) First the total encoding-path gains for the the $A$ and $B$ signals are set and balanced for each PSD, with the PSDs illuminated with neutrons from a strongly scattering sample placed in the beam or from a neutron source placed in the sample position or near the PSDs. Pcwering only the A preamplifiers, the PSD Encoder modules are operated in pulse-height mode, and the gain of each $A$ input is adjusted to give the desired pulseheight spectrum. Similarly, powering only the B preamplifiers, the gain of each B input is adjusted to give a similar pulse-beight spectrum. Next the lower discriminator levels are adjusted to cut off the pulse height spectra at the desired level with both $A$ and $B$ preamplifiers powered and with the PSD module still in pulse-height mode. Live displays in various formats show the pulse-beight spectra as they are being acquired, facilitating all of these adjustments. An example of such pulse-height data at this stage is shown in Fig. 7. Finally, the PSD Encoder modules are switched to

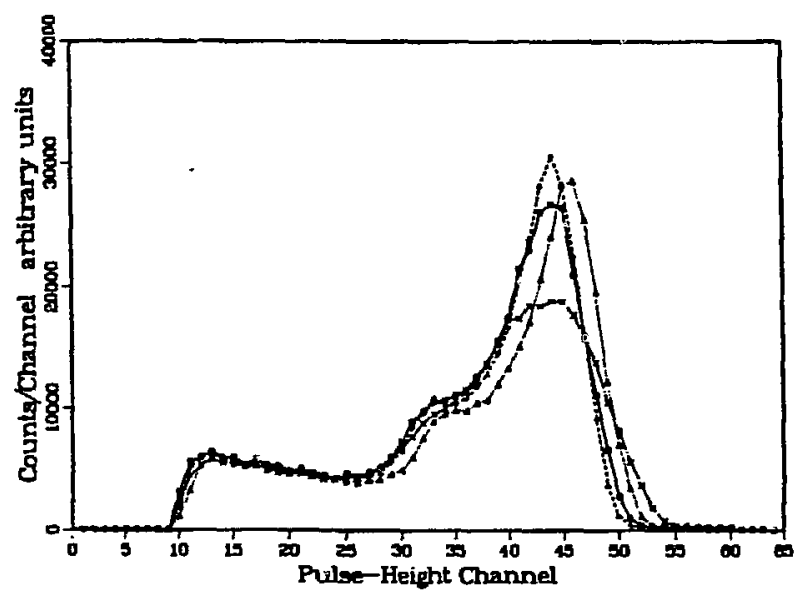

Figure 7. Pulse-height data simultaneously collected from four different PSDs using a PuBe neutron source, after the initial PSD adjustments have been performed. These variations among PSDs are typical.

position mode and position spectra are acquired with the absorbing bar at two different positions $(-1 / 4$ and $-3 / 4$ of the PSD length) in front of the PSDs, while the detector module is more-or-less uniformly illuminated with neutrons. This last step is repeated while adjusting the "Offset" and "Span" controls on the PSD modules until the shadow of the bar shows up in the same position channels on all PSDs. Again, appropriate live graphical displays are available to facilitate this adjustment. Figure 8 shows such position calibration data for several PSDs for one position of the absorbing bar. 


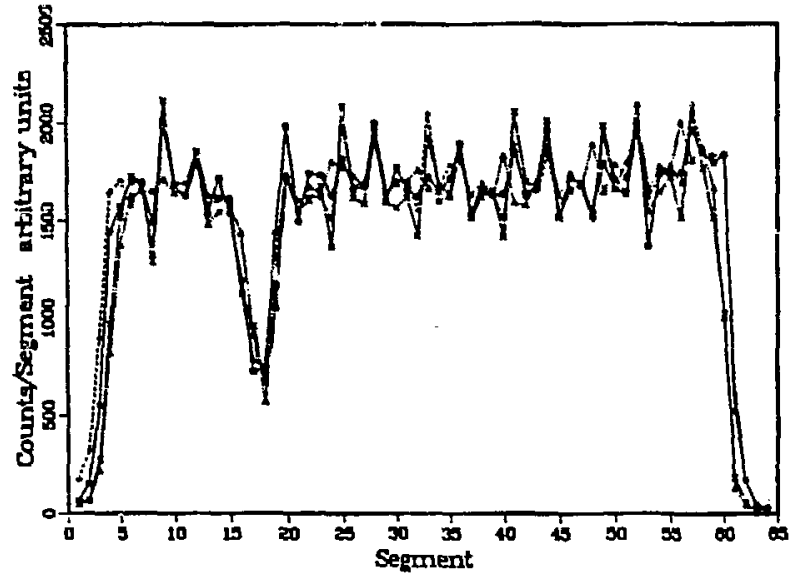

Figure 8. Position data simultaneously collected from four lifferent PSDs, using a PuBe neutron source, after the initial PSD adjustments have been performed. The absorbing bar was positioned in front of the PSDs, and its shadow is seen to be in the same position on all four. The variation of intensity with channel for channels away from the bar position is due to irregularities in channel widths introduced by the limited precision of the analog-to-digital converters.

\section{Detemination of the Absolute Channel-to-Position Mapping}

This procedure should follow the initial adjustment, and should also be repeated occasionally as a check against drifts of the position encoding. A smoothly-varying known distribution of neutrons (a uniform distribution is best) is required, and this can again be provided by a strongly scattering sample in the beam or by a neutron source at the sample position. Position spectra are then recorded for all PSDs with the absorbing bar in several different known positions. These data are least-squares fit to determine the position resolution and the physical positions corresponding to the boundaries of each encoded position element for each PSD, and the results are then stored in an instrument calibration file for subsequent use by the data analysis routines. Since the absorbing bar is driven under computer control, both the data collection and the analysis portions of this procedure can be carried out automatically whenever desired, so long as a suitable neutron source is available.

\section{Automatic Monitoring of Calibration Drifts}

Since the full position calibration described above is fairly time-consuming, and since a sufficiently uniform neutron source is not always available for this purpose, another procedure has been developed for rapid monitoring of calibration drifts. This procedure can be carried out in the absence of neutrons, but does require a suitable file of basic data with which comparisons can be made. This information base consists of three parts, which should be regenerated immediately following the initial adjustment procedure, whenever it is performed. First, a record of all the individual encoding-path gains (each end of each PSD) is obtained by powering each preamplifier bank (A or B), one at a time, injecting test pulses of known magnitude, and recording the pulse-height channels which accumulate these pulses for each PSD. Second, a record of discriminator settings is obtained for each PSD by powering both preamplifier banks, injecting test pulses spanning a range of amplitudes into one preamplifier bank, and recording for each PSD the lowest testpulse amplitude which is accumulated. Finally, an indication of the relative position mapping is obtained for each PSD by powering both preamplifier banks, injecting test pulses of different known amplitudes into banks $A$ and $B$, and recording for each PSD the position channels into which these events are accumulated.

Once this file of basic information is available, drifts in position encoding can be detected easily and automatically by repeating a measurenent of the relative position mapping data (the third set of measurements in the information base) and comparing the results with the values recorded in the information base for each PSD. If further testing is desired, either the gain measurements or the discriminator level measurements or both can be repented and the results compared with the values initially stored in the file. This can all be done automatically in a relatively short time with no user intervention, and so can be included as part of a standard startup procedure at the beginning of each experiment or other suitable interval. If any values are found to have shifted by more than the allowed limits, warnings can be displayed and any other desired action (such as refusal to start data acquisition) can be taken.

\section{PERFORMANCE}

A prototype version of GLAD was installed on the GLAD beamline at IPNS in May, 1988. This prototype instrument, shown schematically in Fig. 9, includes a single detector

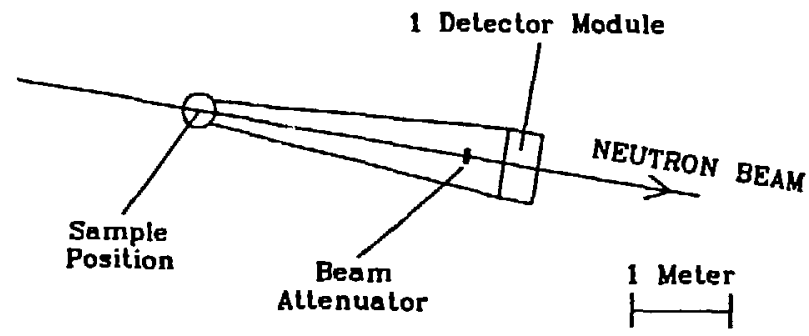

Figure 9. Schematic view of the prototype GLAD instrument.

module containing 55 linear PSDs located in the forwardscattering direction in line with the direct beam. It was built primarily for the development and testing of the destectors, data acquisition, and other technology necessary for the final GLAD instrument, and has been used extensively in developing the procedures for the initial PSD adjustments and calibration as discussed above, as well as to verify that the 
FASTDAS system, the position and time encoding of the the PSDs, and the basic microVax data acquisition software all function satisfactorily. Figure 10 shows an example of one piece of test data collected on this temporary system, indicating the type and quality of data available. The neutron

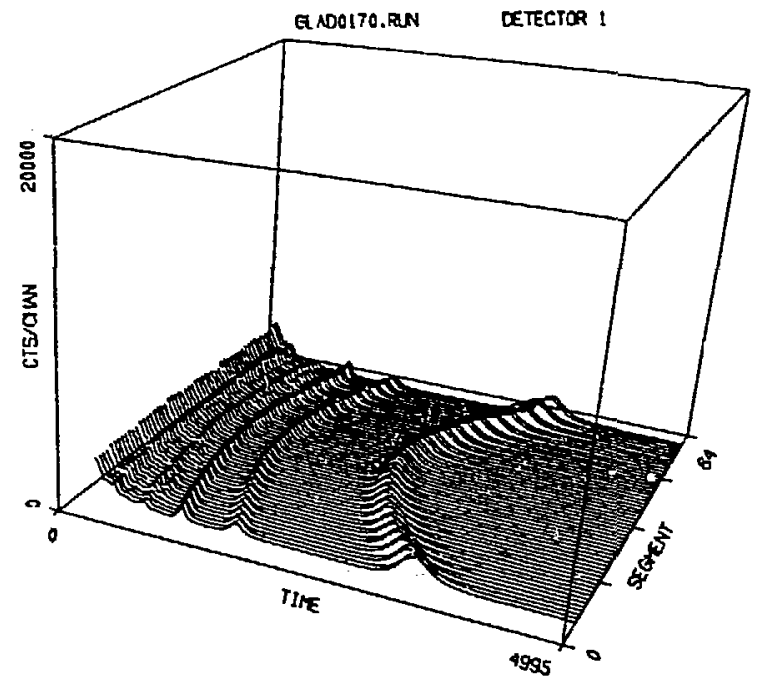

Figure 10. Powder diffraction data from cesium-intercalated graphite, obtained with the prototype GLAD system. Data shown are raw data from a single PSD at the edge of the array, and have been collected with pairs of adjacent segments grouped together. The plot format is one of several available for viewing live histogram data on the instrument microVax. The "time" axis gives TOF in usec.

scattering sample in this case was a crystalline powder of cesium-intercalated graphite, chosen as a test material because of its large interplanar spacings which give rise to Bragg scattering at smal! values of momentum transfer $Q$. The figure shows TOF data from a single PSD located at the edge of the detector array, and the peaks are the constant- $Q$ loci resulting from Bragg scattering from several different interplanar spacings in the sample, each of which leads to scattering at a specific value of $\mathbf{Q}$. Similar spectra were collected concurrently from each of the 55 PSDs in the detector module.

The position-resolution of the position-encoding process has been determined by measuremenis of a neutron flood pattern with the absorbing bar shadowing a region of each PSD, the same type of data required for the position calibration. Least-squares fitting of data such as that in Fig. 8 indicates that the overall neutron position resolution along the PSDs in the current system is $\sim 1.4 \mathrm{~cm}$ fwhm. Repetition of such "bar" measurements has shuwu that the position encoding has remained stable over a period of several months, with shifts in encoded positions on most of the PSDs being less than the detectable limit of $\sim 1 \mathrm{~mm}$.

Initially the PSD encoding produced considerable (as high as 30 percent) integral nonlinearity near the PSD ends. Detailed analysis of this problem[2] predicted that the addition of small resistors between the PSD ends and the preamplifiers would lead to position encoding which was quite linear over the entire PSD length. The data of Fig. 8, collected after 300 $\Omega$ resistors had been added at each end of the PSD, show essentially complete integral linearity as predicted, even at the PSD ends. It was found that $300 \Omega$ was roughly the optimal value for this system (PSD anode resistance $-3500 \Omega$ ). These external resistors bad no measurable effect on the resolution, and so have been incorporated into all PSD encoding paths.

Other measurements with this prototype flight path and detector module have confirmed that the PSDs could be operated satisfactorily in line with the direct beam, with the beam being attenuated only by a $2.5 \mathrm{~cm}$ thick $\mathrm{B}_{4} \mathrm{C} /$ epoxy "beam attenuator" directly in front of the PSDs. Figure 11 shows data from the same sample as for Fig. 10, but this time for a PSD in the middle of the array, and hence centered on the

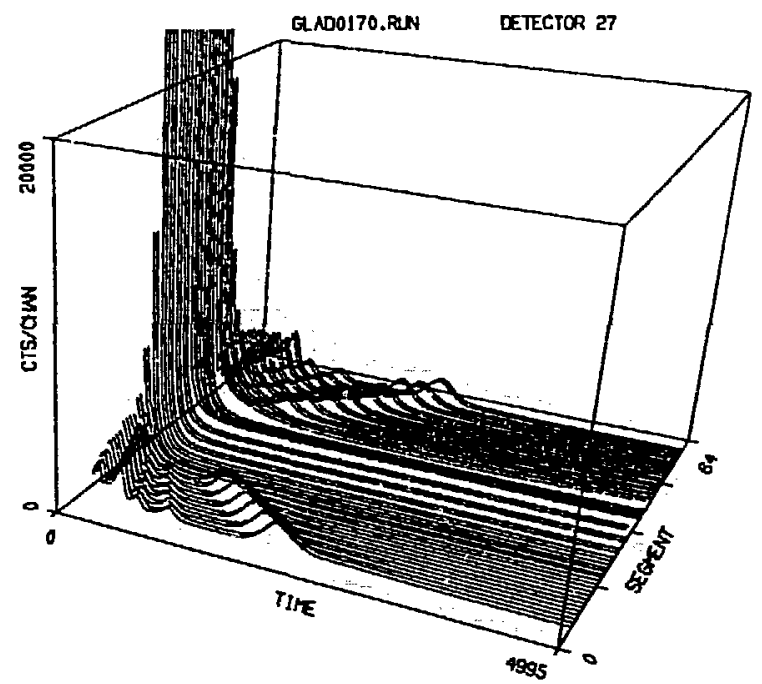

Figure 11. Powder diffraction data coliected at the same time as those of Fig. 10, but from a different single PSD located in the middle of the detector array. See text and Fig. 10 for details.

attenuated direct beam. The shadow of the beam attenuator is clearly seen in the middle segments of the PSD. Also seen in the middle segments is the pattern of fast neutrons in the direct beam, resulting from those neutrons which are not well thermalized in the moderator and bence are of sufficiently high energy to transit the beam attenuator (above several $\mathrm{KeV}$ ). Figure 11 shows clearly the remnants of the prompt pulse at short TOF values and the much lower time-independent level due to delayed fast neutrons which the IPNS target/moderator emits between the prompt pulses. The constant- $Q$ Bragg scattering loci from the same sample interplanar spacings as seen in Fig. 10 can be seen in the portions of the PSD cutside the direct beam and not masked by the attenuator, but they are now shifted to shorter values of TOF (and hence to shorter wavelengths) corresponding to the smaller scattering angles associated with the position segments in this PSD. With the preamplifiers initially used, detector recovery problems were apparent for those PSDs in line with the direct beam at TOFs shorter than $500 \mu \mathrm{sec}$ after the very intense prompt pulse, and 


\section{Detection area}

Position elements

Position resolution

TOF resolution

Deadtine per PSD

Maximum instantaneous rate

(for 10 percent deadtime)

Maximum time-averaged rate
$60 \mathrm{~cm} \times 1.25 \mathrm{~cm}$ per PSD, maximum of 640 PSDs

64 per PSD, maximum of 40,960 elements

$1.4 \mathrm{~cm}$ fwhm along PSD axes, $0.8 \mathrm{~cm}$ normal to PSD axes

$0.5 \mu \mathrm{sec}$

$8 \mu \sec$

12,500 events/sec/PSD on each PSD, with no correlation between individual PSDs

300,000 events/sec some small recovery effects were spparent at TOFs out to $5000 \mu \mathrm{sec}$. Use of faster preamplifiers with output clamping eliminated these effects, and as can be seen by comparing Figs. 10 and 11 it now appears that the PSD is is live with thic direct beam will provide neutron scattering data of a quality comparable to that from the other PSDs (for position segments not shadowed by the beam attenuator).

Tests with "data" from a pulse generator indicated that the FASTDAS histograming board has a maximum time-averaged histograming rate of $\sim 300,000$ events/sec. Measurements with a strongly scattering sample showed that "real" randomized data from this detector module could be collected and histogramed at a time-averaged rate at least as high as $-100,000$ events/sec, the maximum rate which could be produced by this particular sample, with no problems. In this latter case the spectral output of the pulsed neutron source and the TOF of the neutrons to the detector module produced a pronounced time structure in the data rate, so that the instantaneous rates at some of the PSDs were far in excess of the time-averaged rate. Thus this also provided a good demonstration of the ability of the system to cope with high instantaneous rates on individual PSDs.

Table I summarizes the performance, anticipated or verified, for this neutron detection system. All portions of the system are already close to meeting the design goals set forth earlier ir this paper. However several additional development steps are underway to bring the position encoding portions up to the desired level of performance. New preamplifiers are being developed to improve the PSD position resolution from $-1.4 \mathrm{~cm}$ fwhm to $\sim 1.0 \mathrm{~cm}$ fwhm while retaining or increasing the encoding speed. Faster position encoding in the PSD Encoder modules is also desired to reduce the encoding 'eadtime below the present $8 \mu \mathrm{sec}$ per event per PSD. Analog-to-digital converters having greater differential linearity than those presently available are also desirable to reduce or eliminate the -10 percent inequalities in the position widths of the encoded position channels which are so evident in Fig. 8. The $300 \mathrm{KHz}$ histograming speed is already adequate for all but the most demanding situations anticipated, but ma" need to be incrgased somewhat by the time the full complement of PSDs is installed.

\section{ACKNOWLEDGMENTS}

The authors would like to thank M. Faber for his work with the GLAD detector systems, and D. Motitague for allowing his unpublished test data to be used in Figs. 10 and 11. Discussions with R. Berliner and D. F. R. Mildner at the University of Misscuri were very helpful in the initial design of the detection electronics and the calibration procedures.

\section{REFERENCES}

[1] R. K. Crawford, D. L. Price, J. R. Haumann, R. Kleb, D. G. Montague, J. M. Carpenter, S. Susman and R. J. Dejus, "GLAD: the IPNS Glass, Liquid, and Amorphous Materials Diffractometer", in Proceedings of the Tenth Miseting of the International Collaboration on Advanced Neutron Sources, Los Alamos, NM, October 1988, in press.

R. K. Crawford and J. R. Haumann, unpublished infomation, 1989.

[3] R. B. Owen and M. L. Awcosk, "One and Two Dimensional Position Sensing Semiconductor Detectors", IEEE Trans. Nucl. Sci., vol. NS-15, pp. 290-303, June 1968.

[4] J. L. Alberi and V. Radeka, "Position Sensing by Charge Division",IEEE Trans. Nucl. Sci., vol. NS-23, Pp. 251-258, February 1976.

[5] J. R. Haumann, R. T. Daly, T. G. Worlton and R. K. Crawford, "IPNS Distributed Processing Data Acquisition System", IEEE Trans. Nucl. Sci., vol. NS-29, pp. 62-66, February 1982.

[6] J. R. Haumann and R. K. Crawford, "Multiprocessor Data Acquisition System", IEEE Trans. Nucl. Sci., yol. NS-34, pp. 948-953, August 1987.

[7] R. Berliner, D. F. R. Mildiner, O. A. Pringle and J. S. King, "A Large Area Position Sensitive Neutron Detector", Nucl. Instrum. Methods, vol. 185, pp. 481-495, June 1981. 